\title{
A Brief History of Breast Reconstruction and a Discussion of Two Common Autologous Breast Reconstruction Surgeries: The Free Deep Inferior Epigastric Perforator Flap and the Free Transverse Rectus Abdominis Musculocutaneous Flap
}

\author{
Timothy J. Phillips', Jason Williams ${ }^{2}$ \\ ${ }^{1}$ Class of 2012, Faculty of Medicine, Dalhousie University \\ ${ }^{2}$ Division of Plastic Surgery, Dalhousie University, Halifax, Nova Scotia
}

\begin{abstract}
Breast cancer has long been recognized as a challenging disease to treat. In the past 100 years the five-year survival of the disease has increased from a dismal $4-30 \%$ up to $87 \% .1,2$ Surgical techniques have advanced from barbaric removal of the breast in the 15th and 16th century to advanced, highly technical tumour excisions with breastreconstruction involving artificial implants or tissues from the patient's own body. The Deep Inferior Epigastric Perforator Flap (DIEP) and the Transverse Rectus Abdominis Musculocutaneous (TRAM) Flap are two commonly used autologous free flap techniques. Evolution of technique has led to both procedures having high success rates and low complication rates. Whether a DIEP is performed over a TRAM typically depends on surgeon experience and patient anatomy. Selection of which flap to use and whether there are any clear advantages of DIEP over free TRAM is an ongoing debate. Common complications for the free TRAM flap are mainly at the abdominal donor site while the DIEP complications are in the flap itself. Current studies suggest that the three most important factors in selecting a flap are patient's obesity, patient's arterial anatomy at the donor site, and whether a bilateral flap is being performed. Ideally, a multi-center study would be performed which examines a wide range of donor site and flap complications to determine whether the increased time and risk of performing the DIEP equates to better patient outcomes compared to the free TRAM. For now, most authors advocate for proper patient selection, along with intra-operative assessment of the patient's perforating vessels as the best way to optimize outcomes and avoid complications.
\end{abstract}

\section{Introduction}

Breast cancer has long been recognized as a challenging disease to treat. In the past 100 years the five-year survival of the disease has increased from a dismal $4-30 \%$ up to $87 \% \%^{1,2}$ This is due to the combination of advancements in early detection and treatment. Treatment options include surgery, chemotherapy and radiation therapy. ${ }^{2}$ If surgery is one of the treatment options, the patient can be left with a defect in one or both of the breasts. The defect can be repaired using a variety of reconstruction techniques, such as implants or autologous tissue flaps. These reconstruction techniques have advanced along with the treatment options. ${ }^{3}$ This paper will first present a brief history of breast cancer surgery and reconstruction, then focus on the comparison of morbidity between two reconstruction techniques, the free transverse rectus abdominis myocutaneous flap (TRAM) flap and free deep inferior epigastric perforator (DIEP) flap.
History of Breast Cancer Surgery and Reconstruction Breast cancer has been recognized as a fatal disease for thousands of years. Reports of breast cancer have been found in Egyptian papyrus scrolls dating back to 3000 $\mathrm{BC}$ that described it as an incurable disease. The first records of treatment of the disease come from Roman physicians. ${ }^{3}$ The Roman physician Galen reported that breast tumours could be removed and he was one of the first physicians to promote the idea of clear margins for any surgery. ${ }^{3}$ There was little advancement of medicine and surgery through the Dark Ages and it was not until the $15^{\text {th }}$ and $16^{\text {th }}$ century that several different techniques were developed to treat breast cancer. Gerard Tabor developed a scissor-like instrument that could remove an entire breast incredibly quickly, while Dr. Petit of France used a more conservative technique that involved removing the primary tumour and conserving as much of the original breast tissue as possible. ${ }^{2,3}$ 
1 or more large perforators identified?

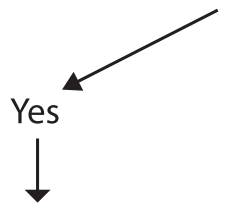

Are these perforators located in the same intermuscular septum?

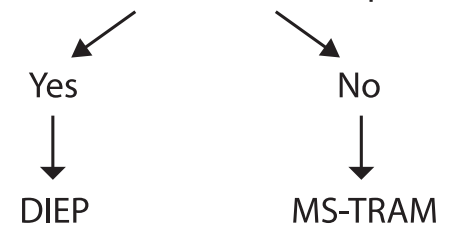

Figure 1. Intra-operative algorithm for choosing DIEP flap vs. Muscle Sparing TRAM ${ }^{19}$

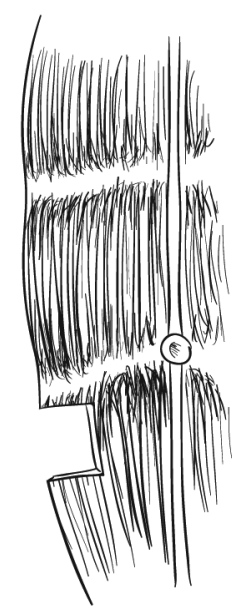

MS 1-M

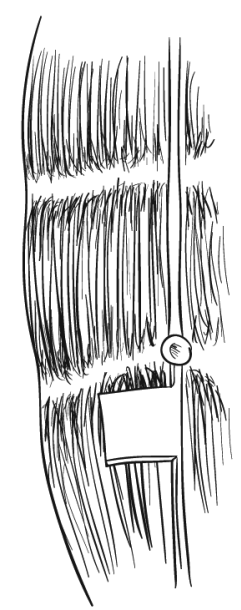

MS 1-L

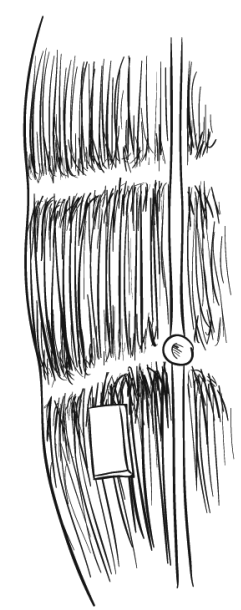

MS 2
Figure 2. Difference in the amount of muscle-sparing between an MS1 flap and an MS2 flap. Figure by AL Martin (2011), adapted from Bahaj et al ${ }^{19}$

It was not until the late 1800's that an American, Dr. William S. Halstead, developed a viable surgical technique. ${ }^{2,3}$ The Halstead technique involved an en bloc resection of the entire diseased breast, the pectoralis major and regional lymph nodes. This technique boasted a $40 \%$ five-year survival rate, compared to other rates of the time, which hovered around $3-30 \%$ five-year survival. ${ }^{2}$ An interesting fact about Halstead was that he was the first to use rubber gloves during a surgical procedure. The gloves were adopted by other members of the surgical team and decreased post-surgical sepsis. ${ }^{3}$

The Halstead technique was the mainstay of advanced surgical treatment of breast cancer. The surgeon Dr. Willy Meyer, had a similar technique to Halstead's that he published at the same time as Halstead. ${ }^{2.3}$ His technique spared more skin allowing for a closure without a skin graft, while the Halstead technique often used a skin graft to close. Dr. Jerome Urban introduced an even more radical mastectomy procedure in the 1950's that also involved removal of mammary vessels and lymph nodes. ${ }^{2,3}$

At the same time the advancements in surgical care for breast cancer were occurring, the utility of radiation therapy for breast cancer was also being studied. A study by the Royal Infirmary in 1948 demonstrated that the combination of radiation therapy and a simple mastectomy could produce a $62 \% 5$-year survival rate, while radical mastectomy at that time had a $42 \% 5$-year survival rate. ${ }^{2}$ These results, combined with the results of future large randomized clinical trials that began taking place in the 1950's have evolved the treatment of breast cancer to what it is today., ${ }^{2,3}$ Currently, the treatment of breast cancer may involve a combination of surgery, radiation and chemotherapy. ${ }^{4}$ Surgery can involve an entire mastectomy or only a lumpectomy depending of the stage and type of cancer. ${ }^{1,2,3,4}$ The Halstead technique is now considered only a surgical procedure of historical importance. ${ }^{2}$

Around the time of Halstead's original publication of his radical mastectomy technique, surgeons in Europe were conducting some of the first post-mastectomy breast reconstructions. ${ }^{5}$ The first recognized reconstruction was performed by Vincent Czerny in 1895, who used a lipoma from the patient's back to replace the breast tissue that he removed due to a fibroadenoma. ${ }^{5}$ Breast reconstruction continued to advance in the early 1900's with many different techniques being attempted including the first latissimus dorsi flap and thoracoabdominal skin flap. ${ }^{5}$ However, reconstruction was abandoned soon after these early attempts. This was mainly due to the Halsteadian school of thought, which considered reconstruction detrimental to the patient's health, ${ }^{5}$ and Halstead famously said "Beware of the man with the plastic operation".

In the 1950's reconstructive breast surgery after breast cancer began to gain popularity and again, many different autologous tissue techniques were used. They included the use of the intact breast as a donor site for tissue, along with the buttocks region, and again the thoracoabdominal area. ${ }^{5}$ It was not until the 1970's when the reconstructive era began to expand with muscle and myocutaneous flaps gaining in popularity. ${ }^{5}$ The benefit of the myocutaneous flap is that it allows the surgeon to move large portions of skin and muscle 
for a single stage reconstruction and often avoid the use of implants. ${ }^{4,5}$

Two of the first myocutaneous flaps that were developed were the latissimus dorsi flap and the transverse rectus abdominis myocutaneous (TRAM) flap. ${ }^{5}$ Both of these techniques are still popular today. The original pedicled TRAM flap has been modified in several ways to give different variations of the procedure which include free TRAM flap, the muscle sparing (MS-1 or 2) TRAM flap, and the DIEP flap. ${ }^{4,5,6}$ The use of microsurgical myocutaneous flaps results in greater total flap survival, reduced donor site morbidity, and increased amount of tissue available for reconstruction. ${ }^{4,5,6}$ Further advancement in flap procedures continued in the 1990's with the deep inferior epigastric perforator (DIEP) flap, which is technically more challenging but is believed to offer decreased donor site morbidity due to preservation of the entire rectus abdominis muscle at the donor site..$^{4,5,7}$

While there are multiple myocutaneous procedures availableforbreastreconstruction, mostreconstructions today use synthetic implants, such as silicone, saline, or mixes of both silicone and saline. ${ }^{4,5}$ While this is not the focus of this review, we address this technique to round out the discussion. Typically an implant is placed after tissue expanders have expanded the chest skin where the breast tissue was excised. The implants are generally implanted beneath the remaining pectoralis major muscle or beneath a latissimus dorsi flap. The benefit of using implants is that it offers a good aesthetic outcome, eliminates the need for external mammary prostheses, with no donor tissue needed and therefore no extra scarring elsewhere in the body. Complications include contraction and distortions along with infection, hematoma, seroma, and skin flap necrosis. $^{4}$

The distinct advantage of using an autologous tissue reconstruction is that the reconstructed breast looks and feels very similar to the natural breast. ${ }^{4}$ Also, there is some evidence to suggest that the autologous graft may be more consistent over time as the implant reconstruction has a higher late revision rate. Disadvantages of the autologous reconstruction is that they are more complex procedures, requiring longer time under anesthetic, additional scarring, abdominal wall morbidities, and risk of flap loss or necrosis. ${ }^{4}$

\section{Description of the TRAM and DIEP flaps}

The TRAM flap is the autologous free tissue transfer breast reconstruction technique that is most used today. ${ }^{5,6}$ The DIEP flap is a newer technique, is more technically demanding than the free TRAM, but is reported to offer reduced donor site morbidity. ${ }^{7}$ Both flaps are similar in that they take the tissue flap from the lower abdomen and use it to reconstruct one or both breasts. The free TRAM flap involves taking skin, adipose tissue and the rectus abdominis muscle and fascia as part of the flap supplied by the deep inferior epigastric artery for the anastamosis at the site of reconstruction. ${ }^{6}$ The DIEP flap spares the rectus abdominis muscle by only taking skin and adipose tissue and dissecting out the deep inferior epigastric artery perforators as they pass through the rectus abdominis muscle. ${ }^{7}$ (Table 1)

The TRAM flap can be performed as a free flap or as a pedicled flap. The free flap utilizes the inferior epigastric arterial system and is completely detached

Table 1. Comparison of TRAM free flaps to DIEP free flaps

\begin{tabular}{|c|c|c|}
\hline & TRAM Free Flap & DIEP Flap \\
\hline Donor site & Lower Abdomen ${ }^{5,6}$ & Lower Abdomen $^{7}$ \\
\hline Tissues used & $\begin{array}{l}\text { 1) skin, } \\
\text { 2) adipose tissue, } \\
\text { 3) Rectus abdominis muscle and } \\
\text { fascia }{ }^{5,6}\end{array}$ & $\begin{array}{l}\text { 1) skin } \\
\text { 2) adipose tissue }\end{array}$ \\
\hline Artery for anastamosis & Inferior epigastric ${ }^{5,6}$ & $\begin{array}{l}\text { Deep inferior epigastric } \\
\text { perforator arteries }^{7}\end{array}$ \\
\hline $\begin{array}{l}\text { Associated } \\
\text { complications }\end{array}$ & Donor site $e^{9-12}$ & Flap loss/ necrosis"11 \\
\hline Overall flap loss & $2 \%{ }^{11}$ & $0.03 \%^{11}$ \\
\hline Post-op pain & Higher than DIEP ${ }^{23}$ & Less than TRAM ${ }^{23}$ \\
\hline Hospital stay & 6 days $^{23}$ & 5 days $^{23}$ \\
\hline $\begin{array}{l}\text { Breast volume to } \\
\text { replace }\end{array}$ & $>1000$ cc's $^{17}$ & $<1000 \mathrm{cc}^{\prime} \mathrm{s}^{17}$ \\
\hline Abdominal obesity & $\begin{array}{l}\text { Mild to Moderate abdominal } \\
\text { obesity better candidates for } \\
\text { TRAM than DIEP17 }\end{array}$ & $\begin{array}{l}\text { Little to no abdominal } \\
\text { obesity preferred for } \\
\text { DIEP Flap }{ }^{17}\end{array}$ \\
\hline $\begin{array}{l}\text { Available arteries for } \\
\text { anastamosis }\end{array}$ & $\begin{array}{l}\text { Does not depend on quality of } \\
\text { perforating arteries }{ }^{17,18,19}\end{array}$ & $\begin{array}{l}\text { 1-2 perforating arteries } \\
\text { at least } 1.5 \mathrm{~mm} \text { in } \\
\text { caliber }^{17,18,19}\end{array}$ \\
\hline
\end{tabular}


from the donor site, while the pedicled flap uses the superior epigastric system and remains attached to the donor site, passing into the site of reconstruction via a subcutaneous tunnel, ${ }^{6}$ thus eliminating the need for microvascular anastomoses. This feature contributes to its ongoing popularity as an option in non-microsurgery breast reconstruction practices. There are also different versions of the TRAM free flap, based on the amount of muscle that is spared during the flap harvest. The MS-1 spares either a strip of medial or lateral muscle, while the MS-2 free flap has the highest degree of muscle sparing of all free TRAM flaps leaving both a medial and lateral portion of muscle. ${ }^{8}$ (Table 1 )

\section{Comparison of results using TRAM and DIEP flap}

Debate has risen over which flap technique offers the best results and whether the increased operative time and demanding technique make it worth striving for a DIEP flap in every case. ${ }^{8}$ Complications that can occur from an autologous breast reconstruction can fall into two broad categories: 1) donor site morbidity and 2) flap loss/necrosis. Donor site morbidity includes such complications as abdominal bulge, laxity, weakness, and abdominal hernia. ${ }^{9,10,11,12,13}$ These complications may be related to the amount of muscle and fascia removed from the abdomen. Flap loss can be broken down into smaller sub-categories, such as total flap loss, fat necrosis, and partial flap loss. ${ }^{11,14,15,16}$ Flap loss is generally related to the level of perfusion within the flap arterial or venous system. The level of perfusion is related to the reliability of the vasculature within the flap and the microvascular anastamoses that connect the flap to the site of reconstruction.

Retrospective studies and meta-analyses have been done to compare free TRAM and DIEP flaps. These studies have demonstrated that free TRAM flaps have a higher degree of donor site morbidity., ${ }^{9,11,112}$ This is due to the TRAM flap harvest removing portions of muscle and fascia, which may result in hernia, bulging, and overall abdominal weakness. The DIEP flap is presumed to have a lower incidence of abdominal morbidities because it does not remove muscle and fascia from the abdomen. (Table 1)

One study demonstrated that approximately $40 \%$ of women who had the TRAM flap reported some difficulties with everyday tasks such as lifting heavy objects and housework, while only $17 \%$ of women who had a DIEP flap reported any problems. ${ }^{10}$ Furthermore, abdominal strength was found to be more greatly impacted in a TRAM flap compared to DIEP flap, however, DIEP flap abdominal strength was decreased compared to normal individuals. ${ }^{9,10}$ DIEP flap patients had half the risk or incidence of developing an abdominal bulge or hernia when compared to TRAM flaps. ${ }^{9,10,11,12}$ The relative risk of developing a hernia or bulge post DIEP flap was 0.49 (95\% Confidence Interval, 0.28-0.86). ${ }^{11}$ Abdominal wall asymmetry was also shown in one study to be lower in DIEP studies compared to the TRAM flaps, with $20 \%$ of TRAM patients having abdominal wall asymmetry compared to only $11 \%$ of DIEP patients. ${ }^{9}$ (Table 1 )

One study comparing the percentage of flap loss in free TRAM versus DIEP flaps has shown that DIEP flaps have twice the risk of overall flap loss (relative risk, 2.05; 95\% CI, 1.16 to 3.61). ${ }^{11}$ For flap complications, there was a statistically significant difference between DIEP and free TRAM flaps in fat necrosis rates $(25.5 \%$ vs. $11.3 \%)$ and total necrosis rates $(4.15 \%$ vs. $1.59 \%, p=0.044) .{ }^{16}$ The reason for the higher degree of flap loss in DIEP flaps is thought to be due to the unreliability of the vasculature within the DIEP flap. TRAM flaps have a lesser degree of total flap loss due the greater reliability of the vasculature within the muscle that is harvested from the abdomen. ${ }^{11,14,15,16}$ One study demonstrated fat necrosis and flap loss percentages in DIEP flaps of 62.5 and 37.5 respectively, compared with the TRAM flaps of 12.9 and 2.2 respectively. This large gap was eliminated when the number of perforating arteries in the DIEP flap was increased. ${ }^{15}$ Venous congestion was monitored in one study and was found to be prevalent in $52 \%$ of failed DIEP cases, but none found in the failed TRAM flaps. This venous congestion could be remedied by using the superficial epigastric vein for additional drainage. ${ }^{14}$ (Table 1 )

\section{Flap Selection}

With apparent opposing benefits and risks between the DIEP and TRAM flap there needs to be selection criteria to decide which flap is used. Nahabedian et al. performed a retrospective study to create selection criteria for the type of flap that should be performed. ${ }^{17}$ The study found that there are three main criteria for choosing a flap technique. These include breast volume to be replaced, abdominal obesity and the number and caliber of perforating arteries within the DIEP flap. ${ }^{17}$ The amount of breast volume that needs to be replaced can be an important factor in deciding whether a TRAM or a DIEP flap should be performed. If the breast volume is greater than 1000 cc's then a TRAM flap should be used, if less than 1000 cc's a DIEP or a TRAM flap can be used. ${ }^{17}$ This is because, as breast volume increases, the number of perforating arteries to vascularize it also increases; if there is insufficient vascularization then necrosis can occur. This was the result observed by Kroll et al., and increasing the number of perforating 
arteries used in the flap prevented the necrosis. ${ }^{15}$ The free-TRAM flap has more perforating vessels than the DIEP flap, therefore, it is preferred for large breast reconstruction. (Table 1)

Morbidly obese patients are generally not candidates for abdominal free flap reconstructions. However, patients with moderate to mild abdominal obesity are better candidates for TRAM flap than a DIEP flap. ${ }^{17}$ The reason once again relates back to the amount of high-quality perforating vessels. The more obese one is, the fewer high-quality perforating arteries are within the tissue. Nahabedian et al. demonstrated that fat necrosis of the flap was dependent on the patient's weight and not dependent on the type of flap used. ${ }^{17}$ Vyas et al. also demonstrated that obesity increases the risk of abdominal morbidity. ${ }^{12}$ (Table 1 )

Even though a specific flap has been planned, this can change once the operation has been started and the patient's abdominal wall vascular anatomy is more apparent. In order for a DIEP flap to be performed there usually has to be one or two perforating vessels with a minimum caliber of $1.5 \mathrm{~mm} \cdot{ }^{17}$ This assessment is made at the level of the anterior rectus sheath after the separation of the flap from the fascia. If this criterion is not met, then the flap may need to be converted to a TRAM flap. ${ }^{17,18}$ One of the most recent studies comparing DIEP and TRAM flaps used an intraoperative algorithm based on availability and quality of each patient's vessels to determine which flap to perform. (Figure 1) The results were that there were no significant differences in intraoperative complications or in minor postoperative complications. However, there was a statistically significant increase in total major postoperative complications in the DIEP study group ( $4 \%$ in DIEP compared to 0\% in TRAM, $\mathrm{p}=0.03$ ). No significant difference was noted in hernia. ${ }^{18,19}$ These results demonstrate the importance of a sufficient arterial system. (Table 1)

The Muscle Sparing TRAM Flap: A Happy Medium? Many of the studies that have been done comparing the DIEP to TRAM flap have failed to mention the type of free TRAM flap that was used. There are 3 different levels of muscle sparing within free TRAM flaps, with the MS-2 version sparing the most. ${ }^{8}$ (Figure 2) The goal of the MS TRAM free flap is to gain the benefits of decreased donor site morbidity associated with DIEP flaps, but to maintain the decreased level of flap loss, and necrosis associated with the non-muscle sparing TRAM flaps. (Table 1)
Four studies were conducted that specifically compared MS-2 TRAM flaps to DIEP flaps. ${ }^{8,12,17,18}$ One study showed that there are no significant differences in fat necrosis, venous congestion, flap necrosis, abdominal bulge, or ability to perform a sit-up after a unilateral DIEP or MS-2 TRAM free flap. ${ }^{8}$ However there was a trend for increased abdominal strength and improved contour after a bilateral DIEP flap compared to a bilateral MS-2-TRAM free flap. ${ }^{8}$ The other three studies demonstrated that there was no difference in abdominal bulging between the MS-2 TRAM free flap and the DIEP flap. ${ }^{12,17,18}$ These results demonstrate that there is little significant difference between the MS-2 TRAM flap and the DIEP flap. (Table 1 and 2)

Three other factors that can be used to compare DIEP to free-TRAM flaps include the cost of the procedures, pain and discomfort, and length of stay in the hospital. Three studies compared the cost of DIEP to TRAM flaps. Two studies demonstrated the DIEP flap is generally less expensive overall than TRAM flaps, while the third study showed no difference between the two. ${ }^{18,20,21,22} \mathrm{~A}$ study by Kroll et al. demonstrated a greater need for post-operative pain medication in TRAM patients compared to DIEP patients, they assumed this represented an increase in pain post TRAM flap compared to DIEP flap. ${ }^{23}$ This study also demonstrated a small but statistically significant decrease in hospital stay ( $<1$ day) post DIEP compared to a TRAM flap $\left(\mathrm{p}=0.026,95 \% \mathrm{CI}\right.$ not provided) ${ }^{23}$ However, the Serletti group did not show any difference in length of hospital stay. ${ }^{18}$ (Table 1$)$

\section{Summary and Conclusion}

There have been many studies comparing DIEP to free TRAM flaps. These studies have consistently showed that DIEP flaps have decreased abdominal wall morbidity compared to TRAM flaps, yet have a higher rate of flap loss and necrosis. The use of the MS-TRAM flap seemed promising to offer a "best of both worlds" flap. However the studies done so far have failed to demonstrate any benefit of this over the DIEP flap. There has also been suggestion by some authors that the rate of flap loss within DIEP flaps will decrease as surgeons become more accustomed to and experienced with the technique. ${ }^{8}$ However, this theory is only something that can be proven in time.

It appears that currently the best way to decide on which flap to use in order to obtain optimal results is based on the patients themselves. According to the results of the studies discussed previously, there are ideal patients for each flap type. The most recent study suggests that vessel anatomy alone is sufficient for 
choosing a flap type. ${ }^{18}$ An intraoperative algorithm is proposed where the decision to perform a DIEP, muscle -sparing, or free TRAM flap is made depending on the perforating vessels present. ${ }^{18,19}$ Also, it was shown that bilateral flaps increase the risk of abdominal wall morbidity; therefore a DIEP flap may be preferential in these women as there is less chance of abdominal wall morbidity.

The criteria for choosing the appropriate flap should be further studied. The DIEP flap was widely accepted as a superior reconstruction option that would replace conventional TRAM flaps at its inception. After the initial fanfare most surgeons consider the DIEP flap to have a place in the flap selection algorithm, but that it is not clearly the best choice for every patient. It appears that proper patient selection, along with intraoperative assessment of the patient's perforating vessels may be the best way to optimize outcomes and avoid complications.

\section{References}

1. Canadian Breast Cancer Foundation. About breast cancer: Breast cancer in Canada 2010. <http://www.cbcf.org/breastcancer/bc whatbc_bc.asp > (12 April 2010)

2. Winchester DP, Trabanino L, Lopez MJ. The evolution of surgery for breast cancer. Surg Oncol Clin N Am 2005;7;14(3):479-98.

3. Cotlar AM, Dubose JJ, Rose DM. History of surgery for breast cancer: Radical to the sublime. Curr Surg 2003;6;60(3):329-37.

4. Reavey P, McCarthy CM. Update on breast reconstruction in breast cancer. Curr Opin Obstet Gynecol 2008;February;20(1):61-7.

5. Losken A, Jurkiewicz MJ. History of breast reconstruction. Breast Dis 2002;12;16(1):3-9.

6. Serletti JM. Breast reconstruction with the TRAM flap: Pedicled and free. J Surg Oncol 2006;94(6):532-7.

7. Granzow JW, Levine JL, Chiu ES, Allen RJ. Breast reconstruction with the deep inferior epigastric perforator flap: History and an update on current technique. J Plast Reconstr Aesthet Surg 2006;6;59(6):571-9.

8. Nahabedian MYMD, Tsangaris TMD, Momen BPD. Breast reconstruction with the DIEP flap or the muscle-sparing (MS2) free TRAM flap: Is there a difference? Plast Reconstr Surg 2005;115(2):436-44.

9. Blondeel PN, Vanderstraeten GG, Monstrey SJ, Van Landuyt K, Tonnard P, Lysens R, Boeckx WD, Matton G. The donor site morbidity of free DIEP flaps and free TRAM flaps for breast reconstruction. Br J Plast Surg 1997;7;50(5):322-30.

10. Futter CM, Webster MHC, Hagen S, Mitchell SL. A retrospective comparison of abdominal muscle strength following breast reconstruction with a free TRAM or DIEP flap. Br J Plast Surg 2000;10;53(7):578-83.

11. Man LMD, M.Sc., Selber JCMD, M.P.H., SerlettiJMMD. Abdominal wall following free TRAM or DIEP flap reconstruction: A metaanalysis and critical review. Plast Reconstr Surg 2009;124(3):75264.

12. Vyas RMMD, Dickinson BPMD, Fastekjian JHMD, Watson JPMD, DaLio ALMD, Crisera CAMD. Risk factors for abdominal donorsite morbidity in free flap breast reconstruction. Plast Reconstr Surg 2008;121(5):1519-26.

13. Wu LCMD, Bajaj AMD, Chang DWMD, Chevray PMMD, Ph.D. Comparison of donor-site morbidity of SIEA, DIEP, and muscle- sparing TRAM flaps for breast reconstruction. Plast Reconstr Surg 2008;122(3):702-9.13.

14. Blondeel PNMD, Ph.D., Arnstein MMD, Verstraete KMD, Ph.D., Depuydt KMD, Van Landuyt KHMD, Monstrey SJMD, Ph.D., Kroll SSMD. Venous congestion and blood flow in free transverse rectus abdominis myocutaneous and deep inferior epigastric perforator flaps. Plast Reconstr Surg 2000;106(6):1295-9.

15. Kroll SS, M, D. Fat necrosis in free transverse rectus abdominis myocutaneous and deep inferior epigastric perforator flaps. Plast Reconstr Surg 2000;106(3):576-83.

16. Sailon AM, Schachar JS, Levine JP. Free transverse rectus abdominis myocutaneous and deep inferior epigastric perforator flaps for breast reconstruction: A systematic review of flap complication rates and donor-site morbidity. Ann Plast Surg 2009;62(5):560-3.

17. Nahabedian MYMD, Momen BPD, Galdino GMD, Manson PNMD. Breast reconstruction with the free TRAM or DIEP flap: Patient selection, choice of flap, and outcome. Plast Reconstr Surg 2002;110(2):466-75

18. Nelson, XA, Guo, Y., Sonnad, SS., Low, DW., Kovach III, SJ., Wu, LC.. Serletti, JM. A comparison between DIEP and muscle-sparing free TRAM flaps in breast reconstruction: a single surgeon's recent experience. Plast Reconstr Surg 2010;126(5): 1428-1435.

19.Bajaj AK, Chevray PM, Chang DW. Comparison of donor-site complications and functional outcomes in free muscle-sparing TRAM flap and free DIEP flap breast reconstruction. Plast Reconstr Surg 2006;117(3):737-46;

20.Kaplan JL, Allen RJMD. Cost-based comparison between perforator flaps and TRAM flaps for breast reconstruction. Plast Reconstr Surg 2000;105(3):943-8.

21.Kroll SSMD, Reece GPMD, Miller MJMD, Robb GLMD, Langstein HNMD, Butler CEMD, Chang DWMD. Comparison of cost for DIEP and free TRAM flap breast reconstructions. Plast Reconstr Surg 2001;107(6):1413-6.

22.Thoma AMD, Veltri KMS, Khuthaila DMD, Rockwell GMD, Duku EMS. Comparison of the deep inferior epigastric perforator flap and free transverse rectus abdominis myocutaneous flap in postmastectomy reconstruction: A cost-effectiveness analysis. Plast Reconstr Surg 2004;113(6):1650-61.

23. Kroll SSMD, Sharma SMD, Koutz CPA, Langstein HNMD, D. Evans GRMD, Robb GLMD, Chang DWMD, Reece GPMD. Postoperative morphine requirements of free TRAM and DIEP flaps. Plast Reconstr Surg 2001;107(2):338-41. 